www.jmscr.igmpublication.org

Impact Factor 5.84

Index Copernicus Value: 83.27

ISSN (e)-2347-176x ISSN (p) 2455-0450

crossref DOI: _https://dx.doi.org/10.18535/jmscr/v5i7.236

Journal Of Medical Science And Clinical Research

\title{
A Clinico-Mycological Study of Human Dermatophytosis in Chitradurga,
} Karnataka, India

\author{
Authors \\ G.L. Aruna ${ }^{1}$ and $^{*}$ Ramalingappa $\mathrm{B}^{2}$ \\ ${ }^{1}$ Assistant Professor, Department of Microbiology, Government Science College, Chitradurga \\ Karnataka, India \\ ${ }^{2}$ Professor, Department of Studies and Research in Microbiology, Davangere University, Davangere, \\ Karnataka, India \\ Corresponding Author \\ Email: ramalingappa.88@gmail.com
}

\begin{abstract}
Dermatophytosis is a superficial fungal infection. It is caused by keratinophilic fungi called dermatophytes. The aim of this work is to detect the most prevalent form of dermatphytosis and the most prevalent dermatophytic fungi from clinical samples of human dermatophytosis. A total of 135 dermatophytosis clinical samples include skin, hair and nail samples were collected from outpatient Department of Dermatology, Government District Hospital, Chitradurga. They were processed by direct microscopy of KOH mount and culture method using Sabouraud Dextrose Agar medium (SDA) with chloramphenicol and Dermatophyte Test Medium (DTM). Among different dermatophyte infections studied, the tinea corporis was most prevalent; it was of $52.68 \%$, followed by tinea cruris $34.40 \%$. The infection was more in men (74\%) than women (26\%). Among four age groups studied, it was more prevalent in 21-40 age group. Culturing and identification of positive clinical samples revealed the presence of dermatophytes like Trichophyton rubrum, Trichophyton mentagrophyte, Epidermophyton floccosum, Microsporum canis and Microsporum gypseum, non dermatophytes such as yeasts like Candida albicans and molds like Aspergillus niger. Among the dermatophytes isolated, the most prevalent species found was Trichophyton rubrum. It constituted $67.74 \%$ of total dermatophyte isolates.
\end{abstract}

Keywords: Dermatophytosis, DTM, KOH mount and Keratinophilic fungi.

\section{INTRODUCTION}

Dermatophytosis (dermatomycosis) is a superficial cutaneous fungal infection of humans and animals caused by dermatophytes. It is thought to be one of the most important public health problems ${ }^{[1]}$. It is also called ringworm or tinea ${ }^{[2]}$. It is ranked as one of the most common cutaneous infection all over the world ${ }^{[3]}$. It is not normally life threatening but it is often difficult to cure completely ${ }^{[4]}$. Dermatophyte infections cause more pain and account for significant costs to society because of its severity, longevity and its resistance to treatment. It is common in tropical countries like India, with high humidity, over population and poor hygienic conditions ${ }^{[5]}$.

Dermatophytes are a group of closely related keratinophilic fungi. They can invade keratinized tissues of humans and animals such as stratum corneum of skin, hair and nails causing dermatophytosis ${ }^{[6]}$. They consist of three genera, Trichophyton, Microsporum and Epidermophyton. They can be identified based on the formation and 
morphology of their conidia ${ }^{[7]}$. Each of which includes several recognized species. Nowadays, 41 species of dermatophytes were identified ${ }^{[8]}$. The $T$. rubrum is the most common superficial fungus accounting for at least $60 \%-80 \%$ of all superficial fungal infections and especially dominant in onychomycosis in humans worldwide ${ }^{[9]}$. These fungi are distributed worldwide with various degrees [6].

Dermatophytes of all ecological types can cause infection in humans. The distribution of dermatophytes varies by region which is affected by factors such as variation in the climate, socioeconomic status, contact with domestic animals and the age of population ${ }^{[10]}$. The infection is more in men than women ${ }^{[9]}$. It has increased during the last decades, particularly among high risk patients, such as the patients with diabetes mellitus, atopia, AIDS and patients undergoing corticosteroid therapy ${ }^{[11]}$. Various studies have been conducted on epidemiology of human dermatophytosis in different parts of this country. Sharma (2012) conducted his study on epidemiology of human dermatophytosis in Jaipur, Balakumar et al., (2012) in Truchirapalli, Tamilnadu, Doddamani et al., (2013) in Gulberga, Karnataka etc., ${ }^{[12,9,13]}$.

Our study focuses on influence of age, gender, climate, unhygienic conditions and socio-economic status of the population on the prevalence of dermatophytosis and study of the most prevalent form of tinea infection and dermatophyte species in the study area.

\section{MATERIALS AND METHODS Study Group}

A total of 135 samples were collected from suspected dermatophytosis patients who were attending Dermatology Department, Government District Hospital, Chitradurga, Karnataka, India. This research involving human subjects is carried out in accordance with the Declaration of Helsinki, as revised in 2013. The clinical symptoms of each case of dermatophytosis were studied and classified according to the type of tinea infection. Detailed case history was collected from dermatophytosis patients with reference to occupation, income, hygienic condition, previous treatment, status of health and contact with dermatophytosis patients, domestic animals and soil. This study was carried out for a period of six months. All 135 clinically suspected dermatophytosis patients were subjected to mycological investigation. The specimens like skin scales, hairs and nails were taken for our study. The samples were collected after the approval of the study by ethical committee.

\section{Collection of Clinical Specimens}

Depending on the site involved, the infected area was cleaned with $70 \%$ alcohol. Gently scraped the surface of the skin at the active margin of the lesion with the help of sterile scalpel and scrapings were collected in a sterilized Kraft paper. The nail clippings were collected from recently invaded nail tissue. Broken or scaly hairs were plucked from the base using sterile forceps. The collected specimens were used for microscopic observation and culture.

\section{Direct Microscopic Examination}

All 135 clinical samples collected were subjected to direct microscopic examination of $\mathrm{KOH}$ mount as shown in Fig. 2. A clean glass slide was taken and a drop of $\mathrm{KOH}$ is placed next to the material. Thoroughly mixed the contents and a cover slip was placed. The preparation was kept in moist chamber and left for 20-60 minutes until softening and digestion of the specimen occurred. Slides were evaluated for the presence of fungal hyphae, arthroconidia or yeast cells under the microscope. Skin scraping samples were processed with $20 \%$ $\mathrm{KOH}$ and hair and nail samples were processed with $40 \% \mathrm{KOH}$.

\section{Culture Study}

All 135 samples were subjected to culture study. The specimens were cultured on SDA medium (with 40 $\mathrm{mg} / \mathrm{L}$ chloramphenicol) and DTM. The culture plates were incubated at $30^{\circ} \mathrm{C}$ for 45 days. The culture plates were observed regularly for the appearance of growth. The samples which showed growth were considered as positive and the colonies were sub cultured on SDA medium containing $40 \mathrm{mg} / \mathrm{L}$ chloramphenicol and $500 \mathrm{mg} / \mathrm{L}$ cycloheximide. The plates which were not showing growth after 45 days of incubation were considered as negative. The colonies were observed for the rate of growth, 
morphology, color (on the surface and reverse) and texture. The fungal culture isolates were identified on the basis of colony morphology as shown in Fig.3 and microscopic observation of wet tease mount preparation of fungal material with lactophenol cotton blue stain. Microscopic observation of nature of hyphae and conidia (macro and micro conidia) helped to differentiate various genera and species as shown in Fig. 4. The dermatophyte test medium (DTM) culture plates were looked for color change of the medium for the confirmation of dermatophytes.

\section{RESULTS}

A total of 135 dermatophytosis patients of different occupation were examined during the study period. The dermatophyte infection was more frequent in socioeconomically poor and unhygenic people, it was about $80 \%$ of the total. Among 135 clinically diagnosed cases of different tinea forms 76 cases were $\mathrm{KOH}$ positive and 59 cases were $\mathrm{KOH}$ negative in direct microscopic examination and 93 cases were culture positive and 42 cases were culture negative is shown in Table 1 . The correlation between results of direct microscopy and culture is shown in Table 2. The prevalence of different forms of dermatophytosis is shown in Table 3. The prevalence of tinea corporis was highest, it was $52.68 \%$, tinea cruris $34.40 \%$, tinea capitis $4.30 \%$, tinea mannum $3.22 \%$, tinea unguium $2.15 \%$ and tinea pedis $2.15 \%$ and lowest was tinea barbae $1.07 \%$. The etiological agents of each type of tinea infection are shown in Table 4. The 93 culture positive samples revealed the presence of dermatophytes like Trichophyton rubrum, Trichophyton mentagrophyte, Epidermophyton floccosum, Microsporum canis and Microsporum gypseum, nondermatophytes like Candida albicans and Aspergillus niger.

Influence of different factors such as age and sex on dermatophyte infection was also studied is shown in Fig, 5 and Fig. 6 respectively. Out of 93 dermatophytosis patients, 74 were males and 26 were females. The dermatophytosis was more prevalent in 21-40 years age group (37\%) and less in 1-20 years age group (15\%).

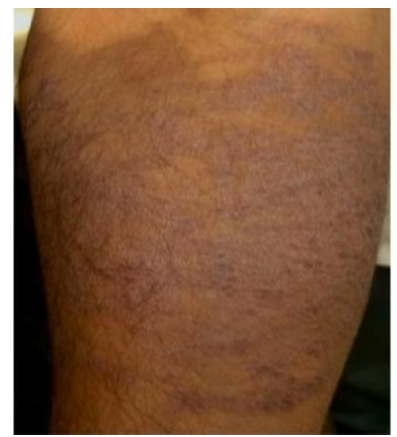

Fig.1a. Tinea corporis

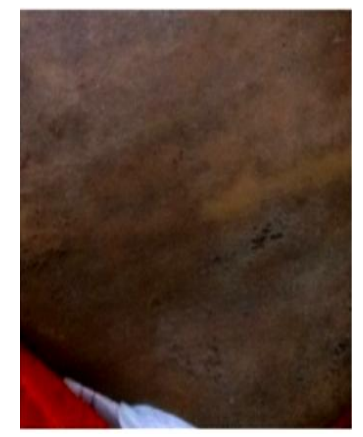

Fig. 1b. Tinea cruris

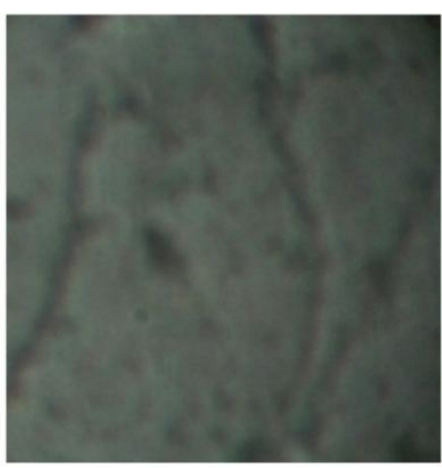

Fig.2 Microscopic view of $\mathrm{KOH}$ mount of Dermatophytosis skin scrapings showing hyphae and conidia

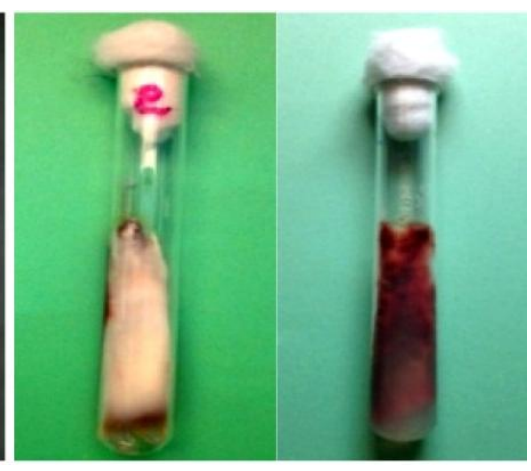

Fig.3 T. rubrum slant culture (a) Obverse (b) Reverse 

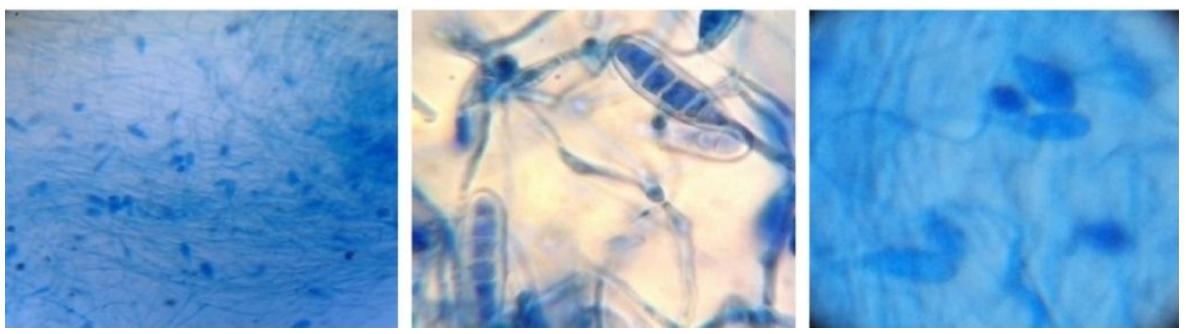

Fig. 4 Microscopic view of lactophenol cotton blue staining showing

(a) T. rubrum microconidia (b) M.canis macroconidia

(c) E. floccosum macroconidia

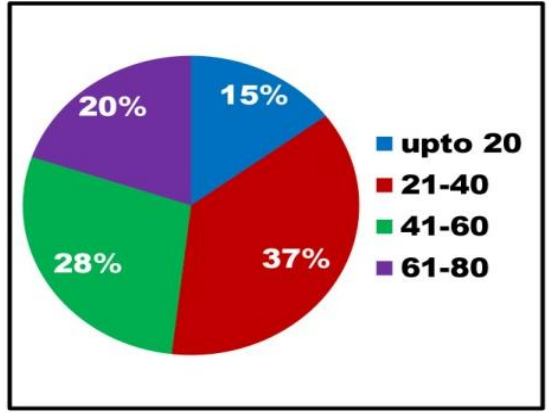

Fig. 5 Dermatophytosis in different age groups

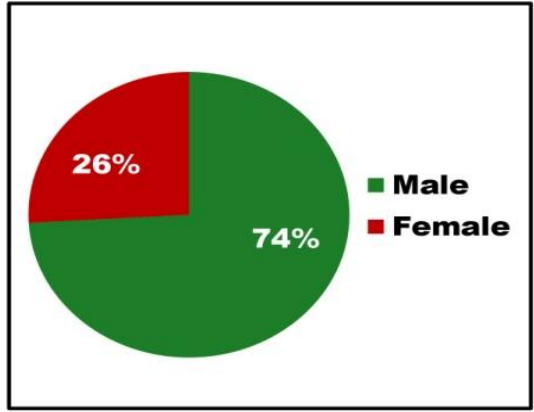

Fig. 6 Sex wise distribution of Dermatophytosis

Table 1. Types of dermatophytosis included in the study and their laboratory results

\begin{tabular}{clccccc}
\hline $\begin{array}{c}\text { Sl. } \\
\text { No. }\end{array}$ & Dermatophytosis & $\begin{array}{c}\text { No. of } \\
\text { Suspected } \\
\text { cases }\end{array}$ & $\begin{array}{c}\text { KOH } \\
\text { Positive }\end{array}$ & $\begin{array}{c}\text { KOH } \\
\text { Negative }\end{array}$ & $\begin{array}{c}\text { Culture } \\
\text { Positive }\end{array}$ & $\begin{array}{c}\text { Culture } \\
\text { Negative }\end{array}$ \\
\hline $\mathbf{1}$ & Tinea corporis & 61 & 39 & 22 & 46 & 15 \\
$\mathbf{2}$ & Tinea capitis & 08 & 03 & 05 & 03 & 05 \\
$\mathbf{3}$ & Tinea manuum & 08 & 03 & 05 & 04 & 04 \\
$\mathbf{4}$ & Tinea ungium & 02 & 01 & 01 & 02 & 00 \\
$\mathbf{5}$ & Tinea barbae & 03 & 01 & 02 & 02 & 01 \\
$\mathbf{6}$ & Tinea pedis & 07 & 02 & 05 & 05 & 02 \\
$\mathbf{7}$ & Tinea cruris & 46 & 27 & 19 & 31 & 15 \\
& Total & $\mathbf{1 3 5}$ & $\mathbf{7 6}$ & $\mathbf{5 9}$ & $\mathbf{9 3}$ & $\mathbf{4 2}$ \\
\hline
\end{tabular}

Table 2. Correltion between results of direct microscopy and culture

\begin{tabular}{|c|c|c|c|}
\hline & KOH Positive & KOH Negative & Total \\
\hline $\begin{array}{l}\text { Culture } \\
\text { Positive }\end{array}$ & 72 & 21 & 93 \\
\hline $\begin{array}{l}\text { Culture } \\
\text { Negative }\end{array}$ & 04 & 38 & 42 \\
\hline Total & 76 & 59 & 135 \\
\hline
\end{tabular}

Table 3. Distribution of tinea types in 93 dermatphytosis culture positive cases

\begin{tabular}{cllc}
\hline Sl.No. & $\begin{array}{c}\text { Dermatophytosis } \\
\text { type }\end{array}$ & $\begin{array}{c}\text { No. of } \\
\text { cases }\end{array}$ & \% of total \\
\hline 1 & Tinea corporis & 49 & $52.68 \%$ \\
2 & Tinea capitis & 04 & $4.30 \%$ \\
3 & Tinea manuum & 03 & $3.22 \%$ \\
4 & Tinea unguium & 02 & $2.15 \%$ \\
5 & Tinea barbae & 01 & $1.07 \%$ \\
6 & Tinea pedis & 02 & $2.15 \%$ \\
7 & Tinea imbricate & 00 & $0.00 \%$ \\
8 & Tinea cruris & 32 & $34.40 \%$ \\
\hline & Total & $\mathbf{9 3}$ & $\mathbf{1 0 0 \%}$ \\
\hline
\end{tabular}


Table 4. Dermatophyte isolates in different types of dermatophytosis

\begin{tabular}{|c|c|c|c|c|c|c|}
\hline \multirow{2}{*}{$\begin{array}{l}\text { Sl. } \\
\text { No }\end{array}$} & \multirow{2}{*}{ Dermatophytosis } & \multicolumn{4}{|c|}{ Dermatophyte Species } & \multirow[b]{2}{*}{ M. gypseum } \\
\hline & & T. rubrum & T. mentagrophyte & E.floccosum & M canis & \\
\hline 1 & Tinea corporis & 35 & 09 & 05 & - & - \\
\hline 2 & Tinea capitis & - & - & - & 03 & 01 \\
\hline 3 & Tinea manuum & 03 & - & - & - & - \\
\hline 4 & Tinea unguium & 01 & 01 & - & - & - \\
\hline 5 & Tinea barbae & - & - & - & 01 & - \\
\hline 6 & Tinea pedis & 02 & - & - & - & - \\
\hline 7 & Tinea imbricate & - & - & - & - & - \\
\hline \multirow[t]{3}{*}{8} & Tinea cruris & 22 & 06 & 04 & - & - \\
\hline & Total & 63 & 16 & 9 & 04 & 01 \\
\hline & Percentage & $67.74 \%$ & $17.20 \%$ & $9.6 \%$ & $4.30 \%$ & $1.07 \%$ \\
\hline
\end{tabular}

\section{DISCUSSION}

The epidemiology of dermatophytosis has substantially changed over the last few years ${ }^{[12]}$. Migration, climatic factors, increased tourism, changes in socioeconomic status, overcrowding, healthcare, environmental cleanliness, personnel hygiene, contact with domestic animals, the age of population and culture may influence the epidemiology of dermatophytoses [14]. The dermatophytosis is more prevalent in people of low socio-economic status and poor personal hygiene ${ }^{[15]}$. Generally, dermatophytes exhibit a cosmopolitan distribution, that is, they are found in different countries of the world with variations in the frequency of particular species ${ }^{[16,17]}$. It occurs in increased frequencies in tropical and subtropical countries ${ }^{[18]}$.

The present study investigated the prevalence of different forms of tinea infections and the most prevalent dermatophyte species in the study area. It also highlighted the distribution of dermatophytosis in different genders and age groups. The dermatophytosis was more prevalent in the chosen study area. The reason could be the selected area is backward district has relatively high population of socioeconomically poor people, mainly consists of farmers and labors. Moreover, climatic condition of the study area is very hot in summer and very cold during winter ${ }^{[19]}$.

The causative agent spectrum of dermatophytosis has markedly changed over the world during the last 100 years ${ }^{[19]}$. The distribution of dermatophytes varies by region ${ }^{[15]}$. In our present study, the most prevalent clinical form found was tinea corporis (Fig 1a) accounts for $52.68 \%$ followed by tinea cruris (Fig 1b), accounting for $34.40 \%$, tinea capitis $4.30 \%$, tinea mannum $3.22 \%$ and tinea pedis $2.15 \%$ of the total cases of dermatophytoses, which was in concordance with studies done by Doddamani in India ${ }^{[13]}$, several epidemiological studies conducted over the last decades have reported that tinea corporis $(35.4 \%)$ is more prevalent followed by tinea cruris $(16.8 \%)^{[20]}$. In our study, dermatophytes were the most common group followed by yeasts and non dermatophyte moulds in the etiology of dermatophytosis which was in agreement with other studies conducted in India ${ }^{[21]}$. The most common isolate obtained in our study was $T$. rubrum (67.74\%). It has been reported that $T$. rubrum (Fig 3) as the most prevalent pathogen of dermatophytosis by many earlier studies ${ }^{[13,20,22]}$, other dermatophytes isolated were $E$. floccosum (16.12\%), T. mentagrophytes (10.75\%), M. canis $(4.93 \%)$ and $M$. gypseum $(1.23 \%)$. The most common yeast isolate in our study was Candida spp, in which $C$. albicans was most frequently reported. The most common nondermatophyte mold isolate in our study was $A$. niger. This was in concordance with studies done by Golia et al. ${ }^{[23]}$.

The age of the person is one of the factors which influence the prevalence of infection among people. In the present study, the disease was more common in age group of $21-40$ years $(37 \%)$ followed by 41 60 years $(28 \%)$. The reason may be due to their active participation in physical activity which results in more sweating and this favours the growth of dermatophytes. The other reason may be, this age 
group people are more social than other age groups, this results spreading of infection among them. This was in concordance with studies done by many workers ${ }^{[23,24]}$. The gender is also one of the factors influence frequency of distribution among population. It was more prevalent in males $(74 \%)$ than females $(26 \%)$. The reason for increased percentage of dermatophytosis in males may be due to their hard physical work and increased exposure to outdoor environment which results sweating, this favours the growth of dermatophytes. This was in agreement with most of the previous studies ${ }^{[9,13]}$.

\section{CONCLUSION}

It may be concluded that hot and humid climate in association with poor socioeconomic status and personel hygiene of the study area may be the major factors responsible for the prevalence of dermatophytosis. In our study, the prevalent tinea form was tinea corporis and the most frequent dermatophyte was T. rubrum. Dermatophytosis can be transmitted quite readily to humans hence, it is important to take appropriate steps to minimize exposure to the fungus. The present study showed the involvement of several fungi in dermatophytosis infection and hence detection of the causative agent is necessary for appropriate treatment of dermatophytosis. If any humans and pet animals in the house develop skin lesions early medical attention should be sought. Early diagnosis and appropriate antifungal therapy is essential for the management of dermatophytosis.

\section{CONFLICT OF INTEREST}

The authors declare that there are no conflicts of interest.

\section{ACKNOWLEDGEMENT}

Authors express sincere thanks to Dr. S.R.D. Vijaya Kumar. Dermatologist and Venerologist, Government District Hospital, Chitradurga, Karnataka for his kind cooperation and guidance in collecting samples from dermatphytosis patients. We would also like to acknowledge the invaluable cooperation of the patients participated in this study.

ETHICS APPROVAL AND CONSENT TO PARTICIPATE
All clinical isolates were processed under the ethical approval of Basaveshwara Medical College Internal Review Board (IRB), Chitradurga, Karnataka, India. All participants signed written informed consent prior to specimen collection and for the publication of this report and any accompanying images.

\section{REFERENCES}

1. Grapple FS, Bishop TC and Blank F. Immunology of Dermatophytes and Dermatophytosis. Bacteriological Reviews. 1974, 38:222-250.

2. Weitzman I and Summerbell RC. The Dermatophytes. Clinical Microbiological Reviews. 1995, 8: 240-259.

3. Ndako JA, Osemwegie OO, Spencer THI, Olopade BK, Yunusa GA and Banda J. Prevalence of Dermatophytes and other associated Fungi among school children. Global Advanced Research Journal of Medicine and Medical Sciences. 2012, 1:4956.

4. Kaufman G, Berdicevsky I, Woodfolk JA and Horwitz BA. Markers for Host-Induced Gene Expression in Trichophyton Dermatophytosis. Infection and Immunity. 2005, 73:6584-6590.

5. Koga $T$, Duan $H$, Urabe $K$, Furue $M$, Maidashi and Higashi-Ku. Immunohistochemical detection of interferon-gammaproducing cells in dermatophytosis. Europian Journal of Dermatology. 2010, 11:105-107.

6. Woodfolk JA. Allergy and Dermatophytes. A Clinical Microbiology Reviews. 2005, 18:30-43.

7. Madhavi S, Rama Rao MV and Jyothsna K. Mycological study of Dermatophytosis in rural population. Annals of Biological Research. 2011,2:88-93.

8. Gharachorlou A, Hashemi SJ, Gharachorlou $\mathrm{S}$ and Ghalekandi JG. Study of relationship among Testosterone and Dermatophytosis due to Microsporium gypseum. Advances in Environmental Biology. 2011, 5:2166-2168.

9. Balakumar S, Rajan S, Thirunalasundari $\mathrm{T}$ and Jeeva S. Epidemiology of dermato- 
phytosis in and around Tiruchirapalli, Tamilnadu, India. Asian Pacific Journal of Tropical Disease. 2012, 2:286-289.

10. Cortez CAA, de Souza JVB, Sadahiro A and de Oliveira JAA. Frequency and aetiology of dermatophytosis in children age 12 and under in the state of Amazonas, Brazil. Revista Iberoamericana de Micologia. 2012, 29:223-226.

11. Zuzarte M, Gonçalves MJ, Canhoto J and Salgueiro, L. Antidermatophytic activity of essential oils. In: A. Mendez-Vilas, editors. Science against microbial pathogens: communicating current research and technological advances. Formatex Spain, 2011. pp 1167-1178.

12. Sharma $M$ and Sharma R. Profile of dermatophytic and other fungal infections in Jaipur. Indian Journal of Microbiology. 2012, 52: 720-740.

13. Doddamani PV, Harshan KH, Kanta RC, Gangane $\mathrm{R}$ and Sunil KB. Isolation, Identification and Prevelance of Dermatophytes in Tertairy Care Hospital in Gulbarga District. People's Journal of Scientific Research. 2013, 6:10-13.

14. Havlickova B, Czaika VA and Friedrich M. Epidemiological trends in skin mycoses worldwide. Mycoses. 2008, 51:2-15.

15. Cortez CAA, de Souza JVB, Sadahiro A and de Oliveira JAA. Frequency and aetiology of dermatophytosis in children age 12 and under in the state of Amazonas, Brazil. Revista Iberoamericana de Micologia. 2012, 29:223-226.

16. Seebacher C, Bouchara JP and Mignon B. Updates on the Epidemiology of Dermatophyte Infections. Mycopathologia. 2008, 166:335-352.

17. Dehghan M, Hajian S, Alborzi N, Borgheyee A and Noohi AH. Clinico-mycological Profiles of Dermatophytosis in Gorgan, North of Iran. Iranian Journal of Dermatology. 2009,12:13-15.

18. Mathur M, Kedia SK and Ghimire RBK. "Epizoonosis of Dermatophytosis": A
Clinico - Mycological Study of Dermatophytic Infections in Central Nepal. Kathmandu University Medical Journal. 2012, 37:30-33.

19. Hayette MP and Sacheli R. Dermatophytosis, Trends in Epidemiology and Diagnostic Approach. Current Fungal Infection Reports. 2015, 9:164-179.

20. Hanumanthappa SB, Sarojini SB and Shilpashree SB, Muddapur SB. Clinicomycological study of 150 cases of dermatophytosis in a tertiary care hospital in South India. Indian Journal of Dermatology. 2012, 57: 322-3.

21. Venkatesh VN and Kotian N. Dermatophytosis: A Clinico-mycological profile from a tertiary care hospital. Journal of International Medicine and Dentistry. 2016, 3:96-102.

22. Kaur R, Kashyap B and Bhalla P. Onychomycosis-epidemiology, diagnosis and management. Indian Journal of Medical Microbiology. 2012, 26:108-16.

23. Golia S, Hittinahalli V, Vasudha CL, Sangeetha L, Mohan KTM and Syrti C. A study on the mycological profile of onychomycosis. Journal of Evolution of Medical and Dental Sciences. 2012, 1:12471255.

24. Ramaraj V, Vijayaraman RS, Rangarajan S and Kindo AJ. Incidence and prevalence of dermatophytosis in and around Chennai, Tamilnadu, India. International Journal of Research in Medical Sciences. 2016, 4:695700. 